\title{
Phosphatidylserine-dependent anti-prothrombin antibodies (aPS/PT) in infliximab-treated patients with inflammatory bowel diseases
}

\author{
Karin Malíčková • Dana Ďuricová • \\ Martin Bortlík · Ivana Janatková · Tomáš Zima • \\ Milan Lukáš
}

Received: 14 October 2012/Accepted: 4 December 2012/Published online: 23 December 2012

(C) Springer-Verlag Italia 2012

\begin{abstract}
Purpose To (1) examine the occurrence and concentrations of aPS/PT and aPL in inflammatory bowel disease (IBD) patients at the beginning of and during anti-TNFalpha therapy with infliximab; (2) investigate the link of the aPS/PT and aPL presence with antibodies to infliximab (ATI) formation; and (3) examine possible clinical consequences of aPS/PT and/or aPL positivity in IBD patients. Materials and methods Thirty (30) IBD patients treated with infliximab were analyzed regarding aPS/PT, aPL, and ATI antibody serum levels by standardized ELISAs at treatment weeks 2 (W2) and 14 (W14).

Results At W2, $40 \%$ of infliximab-treated patients had elevated aPS/PT and $16.7 \%$ had elevated aPL serum levels. At W14, the proportion of aPS/PT-positive sera decreased to $16.6 \%$, whereas aPL distribution remained unchanged. Moreover, concentrations of aPS/PT have shown significant differences at W2 (16.64 [10.06; 33.06] U for IgG and 18.46 [9.18; 32.48] U for IgM) and at W14 $(8.24[2.78 ; 19.82] \mathrm{U}$ for $\operatorname{IgG}$ and 8.57 [5.55; 26.82] $\mathrm{U}$ for $\operatorname{IgM}), p=0.009$ and $p=0.003$, respectively. In ATI-positive samples, aPS/PT IgG were more frequent ( $p=0.001$ for $\mathrm{W} 2$ and $p=0.003$ for W14), whereas aPS/PT IgM and aPL IgG/IgM did not show such association.
\end{abstract}

K. Malíčková $(\bowtie) \cdot$ I. Janatková · T. Zima

Laboratory of Clinical Immunology and Allergology,

Institute of Medical Biochemistry and Laboratory Diagnostics,

1st Faculty of Medicine and General University Hospital,

Charles University in Prague, Karlovo náměstí 32,

12111 Prague 2, Czech Republic

e-mail: kmali@lf1.cuni.cz

D. Ďuricová · M. Bortlík · M. Lukáš

Clinical and Research Center for Inflammatory Bowel Disease,

ISCARE a.s. and Charles University, Prague, Czech Republic
Conclusions Higher concentrations of aPS/PT IgG and IgM were found in IBD patients at the beginning of the biological treatment period compared to the maintenance treatment period. Moreover, aPS/PT IgG were more frequent in ATI-positive individuals, which was not observed in aPL. We speculate that there is a relationship between the aPS/PT and the severity of inflammation and autoaggressive processes in IBD.

Keywords Inflammatory bowel diseases - Crohn's disease - Ulcerative colitis - Phosphatidylserine-dependent antiprothrombin antibodies - Antiphospholipid antibodies . Antibodies to infliximab

$\begin{array}{ll}\text { Abbreviations } \\ \text { ANA } & \text { Antinuclear antibodies } \\ \text { anti-dsDNA } & \text { Anti-double-stranded DNA antibodies } \\ \text { aPL } & \text { Antiphospholipid antibodies } \\ \text { aPS/PT } & \begin{array}{l}\text { Phosphatidylserine-dependent } \\ \text { anti-prothrombin antibodies }\end{array} \\ \text { ATI } & \text { Antibodies to infliximab } \\ \text { CD } & \text { Crohn's disease } \\ \text { IBD } & \text { Inflammatory bowel disease } \\ \text { IFX } & \text { Infliximab } \\ \text { TNFa } & \text { Tumor-necrosis factor alpha } \\ \text { UC } & \text { Ulcerative colitis } \\ \text { W2 } & \text { 2nd week of the treatment } \\ \text { W14 } & \text { 14th week of the treatment }\end{array}$

\section{Introduction}

Inflammatory bowel disease (IBD) is of a group of gastrointestinal inflammatory diseases with two major types: ulcerative colitis (UC) and Crohn's disease (CD). Tumor 
necrosis factor alpha (TNFa) plays an important role in the pathogenesis of IBD. The use of therapeutic TNFa inhibitors has brought about great progress, especially in the treatment of patients with high and persistent IBD activity, complicated disease behavior, and unresponsiveness to other immunomodulatory treatments [1]. Several therapeutic anti-TNFa monoclonal antibodies are currently used in IBD treatment; the most frequently used is infliximab, which is a chimeric monoclonal antibody against $\mathrm{TNFa}$ administered as an intravenous infusion.

In spite of the great advantages of infliximab in IBD treatment, the risk of the development of autoimmune processes related to infliximab administration must be mentioned, ranging from asymptomatic laboratory alterations to life-threatening systemic autoimmune diseases [2, 3]. It is known that infliximab may stimulate the production of different autoantibodies such as antinuclear antibodies (ANA), anti-double-stranded DNA antibodies (anti-dsDNA), antiphospholipid antibodies (aPL), and antibodies to infliximab (ATI). Various workgroups have shown that infliximab led to the development of ANA in 29-77\%, anti-dsDNA antibodies in 10-29\%, aPL in 21-27\%, and ATI in 7-19\% of patients treated with infliximab $[4,5]$.

Patients with IBD are at increased risk for thrombotic complications, which are threefold more likely in IBD patients than controls, and the relative risk exceeds 15 -fold during disease flare-ups [6-9]. Generally, an inflammatory systemic state can lead to endothelial dysfunction, secondary dyslipidemia, and activation of coagulation [10, 11]. IBD patients could be at higher risk of vascular thrombosis as well through induction of thrombophilic autoantibodies, especially aPL.

We have focused on the antibodies against phosphatidylserine/prothrombin complexes (antiphosphatidylserinedependent antiprothrombin antibodies, aPS/PT), which are a subgroup of antiphospholipid antibodies and may be associated with vascular thrombosis and positivity of lupus anticoagulant phenomena [12-15]. Since there are scanty published data concerning prothrombotic variables in active IBD patients and only a few published studies analyzing such patients treated with TNFa antagonists, we have examined (1) the incidence of aPS/PT in IBD patients with active disease;

(2) the behavior of aPS/PT development during anti-inflammatory treatment with the anti-TNFa blocker infliximab; and (3) the clinical consequences of pertinent aPS/PT positivity with thrombotic events in examined IBD patients.

\section{Materials and methods}

We have conducted a study of aPS/PT, aPL, and ATI serum levels in 30 randomly selected IBD patients treated with infliximab.
Patients

The cohort of patients comprised 15 females and 15 males aged 21-63 (median 33 years) previously naïve to antiTNFa therapy and treated with infliximab. Eighteen (18) of the participants suffered from Crohn's disease, and 12 had ulcerative colitis with moderate to severe activity.

Blood samples were collected during the induction therapy at week 2 (W2, after the first and before the second infliximab infusion) and during the maintenance treatment at week 14 (W14). Blood samples were taken from the cubital vein. Clotted blood samples were centrifuged for $10 \mathrm{~min}$ at ambient temperature; $1,300 \mathrm{~g}$ and separated serum aliquots were frozen at $-80{ }^{\circ} \mathrm{C}$ and placed in the IBD serum bank. The frozen serum samples were thawed once on ice before analysis.

Interventions

IBD patients enrolled in the study were treated according to standard clinical practice as a scheduled strategy. Infliximab treatment using a dose of $5 \mathrm{mg} / \mathrm{kg}$ of body weight was started in the induction phase, using three intravenous infusions in weeks 0,2 , and 6 . After that, if a response was achieved, maintenance therapy was continued every other month. A standard assessment of disease activity before and after the induction period was done, including clinical markers and laboratory examinations.

Immunosuppressants such as azathioprine or 6-mercaptopurine were taken by 11 of the 30 patients (37\%); additionally, corticosteroids were taken by 16 of the 30 patients $(53 \%)$ and mesalazine by 21 of the 30 patients $(70 \%)$.

\section{Ethical aspects}

The study was approved by the Institutional Ethical Committee. The purpose and procedures of the study were explained to participants, who signed informed consent forms.

Laboratory evaluation

Serum aPS/PT, aPL, ATI levels and fecal calprotectin were measured by standardized ELISAs. Serum C-reactive protein (CRP) was detected by immunonephelometry.

aPS/PT IgG and IgM were detected by QUANTA Lite ${ }^{\circledR}$ aPS/PT IgG and QUANTA Lite ${ }^{\circledR}$ aPS/PT IgM (INOVA Diagnostic Inc., San Diego, USA) by the sandwich ELISA technique. Briefly, sera were pipetted to the plastic microwell plate wells coated with purified PS/PT complex. Upon incubation, unbound protein was removed by washing, and anti-human IgG or IgM horseradish peroxidase (HRP) 
labeled conjugate was added to the wells. After further incubation and washing, a peroxidase substrate was added and the enzymatic production was stopped. The presence or absence of aPS/PT antibodies was determined spectrophotometrically at $450 \mathrm{~nm}$ using a MRXII (Dynatech, UK) photometer and analyzed using the software Revelation (Dynatech, UK). The reference range 0-30 Units was used per the manufacturer's recommendations.

Detection of aPL was accomplished by anti-phospholipid screen IgG/IgM (Orgentec, Mainz, Germany). Serum samples with elevated values $\geq 10$ GPLU/MPLU were further investigated for $\operatorname{IgG}$ and $\operatorname{IgM}$ class autoantibodies against beta-2-glycoprotein I, cardiolipin, phosphatidylserine, phosphatidylinositol, and phosphatidic acid using standardized enzyme-linked immunosorbent assay (ThromboCombo, Orgentec, Mainz, Germany).

ATI were detected by enzyme-linked immunosorbent assay with the use of antibody to Infliximab Q-ATI ELISA Quantitative Analyses (Matriks Biotek, Ankara, Turkey). Q-ATI is a sandwich assay for the determination of antibodies against infliximab in serum and plasma samples. The reference range $0-8 \mathrm{ng} / \mathrm{mL}$ was used based on our own lab reference ranges using data from our own equipment and donors' sera.

Systemic inflammation was assessed by CRP serum levels (Dade Behring High Sensitivity CRP, Siemens Medical Solutions Diagnostics, Erlangen, Germany). Local inflammation of the intestinal mucosa was assessed with the help of fecal calprotectin measurement (EK-CAL ELISA Bühlmann, Schönenbuch, Switzerland).

\section{Statistical analyses}

Statistical analysis was performed using the software Statistica CZ 10.0 (StatSoft Inc, Tulsa, USA). Different groups were compared using the Mann-Whitney $U$ test or a two-sided Kruskal-Wallis non-parametric test. The Spearman Rank Correlation Test was used to identify correlations between variables. The threshold for significance was set at $p<0.05$.

\section{Results}

Basic descriptive characteristics of examined cohorts are presented in Table 1.

At the beginning of the infliximab treatment at W2, $38.7 \%$ of infliximab-treated patients had elevated aPS/PT. Three (3) aPS/PT positivities were found simultaneously in IgG and IgM isotypes; nine (9) patients had solitary aPS/ PT IgG.

W2 was characterized with weak to moderate aPL positivity in $16.1 \%$ of patients. In one (1) case aPL IgG
Table 1 Descriptive characteristics of examined cohorts

\begin{tabular}{lc}
\hline Gender $n(\%)$ & \\
F & $15 / 30(50 \%)$ \\
M & $15 / 30(50 \%)$ \\
Age, median (IQR) & $33(29 ; 44)$ \\
Diagnosis $n(\%)$ & \\
CD & $18 / 30(60 \%)$ \\
L1 & $8 / 18(44 \%)$ \\
L2 & $2 / 18(12 \%)$ \\
L3 & $8 / 18(44 \%)$ \\
B1 & $6 / 18(33 \%)$ \\
B2 & $7 / 18(39 \%)$ \\
B2 +3 & $4 / 18(22 \%)$ \\
B3 & $1 / 18(6 \%)$ \\
UC & $12 / 30(40 \%)$ \\
E2 & $4 / 12(33 \%)$ \\
E3 & $8 / 12(67 \%)$ \\
Concomitant treatment $n(\%)$ & \\
Immunosuppressants & $11 / 30(37 \%)$ \\
Corticosteroids & $16 / 30(53 \%)$ \\
Mesalazine & $21 / 30(70 \%)$ \\
Response to the IFX treatment $n(\%)$ & \\
Responders & $20 / 30(67 \%)$ \\
Responders with adverse events & $6 / 30(20 \%)$ \\
Primary non-responders & $1 / 30(3 \%)$ \\
Secondary non-responders & $3 / 30(10 \%)$ \\
\hline
\end{tabular}

$F$ females, $M$ males, $I Q R$ interquartile range, $I F X$ infliximab, $C D$ Crohn's disease, $L 1$ ileal location of $C D, L 2$ colonic location of $C D$, $L 3$ ileo-colonic location of $\mathrm{CD}, B 1$ non-stricturing non-penetrating behavior of $\mathrm{CD}, B 2$ stricturing behavior of $\mathrm{CD}, B 3$ penetrating behavior of $\mathrm{CD}$ [16], UC ulcerative colitis, E2 left-sided UC, E3 extensive UC [16]

were detected and in four (4) patients aPL IgM were found; none of these individuals has shown double $\operatorname{IgG}$ and $\operatorname{IgM}$ positivity.

At W14, proportion of aPS/PT-positive sera decreased approximately by half to $16 \%$, whereas aPL distribution remained unchanged, see Table 2.

Moreover, concentrations of aPS/PT have shown significant differences at W2 compared to W14 for both isotypes examined, see Fig. 1.

Interestingly, in both $\mathrm{W} 2$ and W14 cohorts, simultaneous aPS/PT and aPL positivity was not found.

ATI were found in $2 / 30(6.7 \%)$ patients after the first infliximab infusion at W2, and in 6/30 (20\%) patients during W14 of the maintenance treatment, see Table 2.

More frequent were aPS/PT IgG and their serum levels were significantly higher in ATI-positive samples (see Fig. 2a, b), whereas aPS/PT IgM and aPL IgG/IgM did not shown such association. 
Table 2 aPS/PT IgG/IgM, aPL IgG/IgM, and ATI IgG during the anti-TNFa treatment of 30 IBD patients with infliximab

\begin{tabular}{lll}
\hline & W2 $n(\%)$ & W14 $n(\%)$ \\
\hline aPS/PT IgG & $9(30 \%)$ & $3(10 \%)$ \\
aPS/PT IgM & 0 & 0 \\
aPS/PT IgG and IgM & $3(10 \%)$ & $2(6.7 \%)$ \\
aPL IgG & $1(3.3 \%)$ & $1(3.3 \%)$ \\
aPL IgM & $4(13.3 \%)$ & $4(13.3 \%)$ \\
aPL IgG and IgM & 0 & 0 \\
ATI IgG & $2(6.7 \%)$ & $6(20 \%)$ \\
\hline
\end{tabular}

aPS/PT phosphatidylserine-dependent anti-prothrombin antibodies, $a P L$ antiphospholipid antibodies, ATI antibodies to infliximab, W2 second week of the biological treatment, W14 14th week of the biological treatment

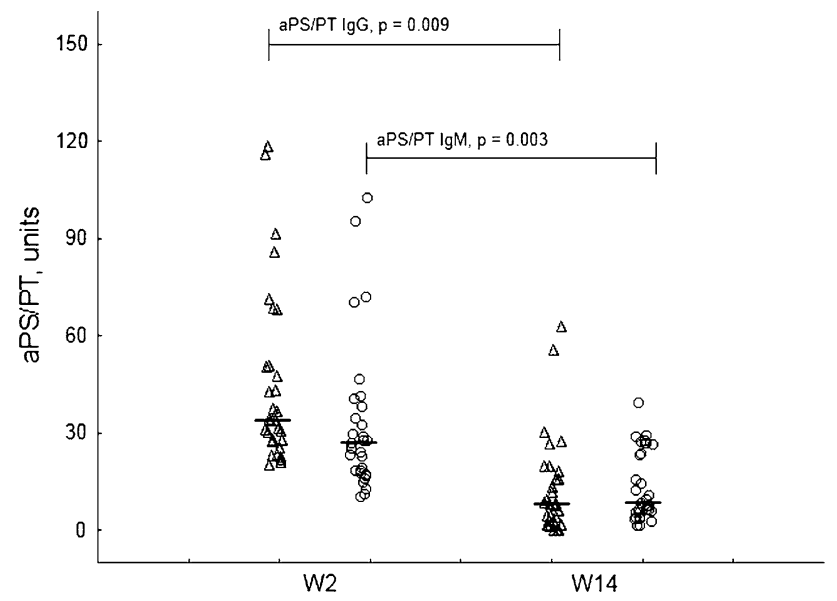

Fig. 1 Development of aPS/PT serum concentrations during IBD treatment with infliximab. $a P S / P T$ phosphatidylserine-dependent antiprothrombin antibodies, W2 second week of the biological treatment, W14 14th week of the biological treatment

None of the autoantibodies monitored have shown dependence on systemic inflammation measured by serum CRP or on local intestinal inflammation measured by fecal calprotectin.

Analyses did not identify age, gender, diagnosis ( $\mathrm{CN}$ or $\mathrm{UC}$ ), or concomitant immunosuppressive treatment as a risk factor for developing aPS/PT.

None of the aPS/PT and/or aPL-positive IBD patients experienced clinical symptoms of antiphospholipid syndrome such as vascular thrombosis or miscarriage either before or during the biological treatment of IBD with infliximab.

In summary, aPS/PT concentrations have shown descending tendency during the biological treatment of IBD with infliximab and displayed different behavior compared to aPL and ATI; aPS/PT IgG were significantly more frequent in patients with ATI positivity.
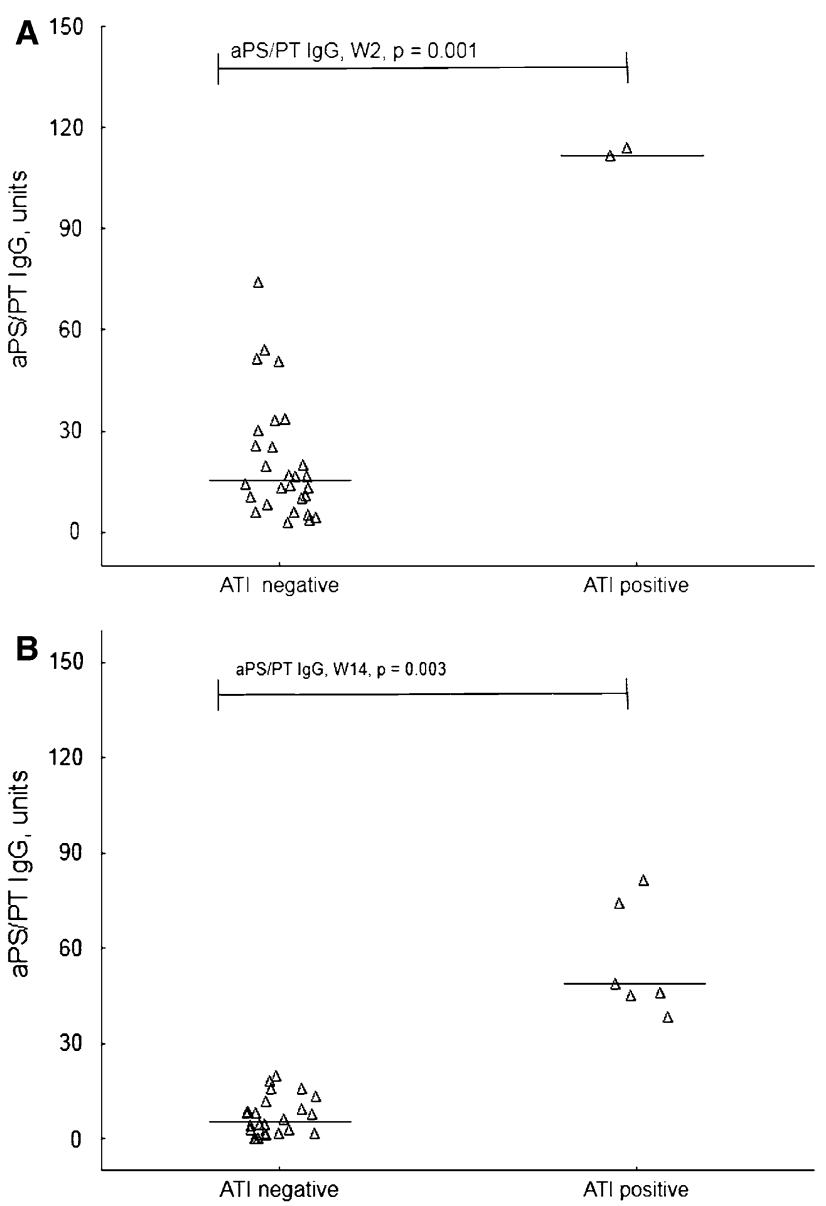

Fig. 2 aPS/PT antibodies in ATI negative and ATI-positive sera. a W2 b W14. aPS/PT phosphatidylserine-dependent antiprothrombin antibodies, ATI antibodies to infliximab, W2 second week of the biological treatment, W14 14th week of the biological treatment

\section{Discussion}

To our knowledge, this is the first report arising from the investigation of serum aPS/PT antibodies in IBD patients during immunomodulatory treatment with anti-TNFa blocker. It shows a relatively high incidence of aPS/PT $(40 \%)$ in patients with active disease where biological treatment was needed and consequent decreasing of aPS/ PT levels due to the anti-inflammatory effect of infliximab.

This behavior is different from "classic" aPL and to other organ non-specific autoantibodies observed in numerous studies $[2-5,17,18]$. It is known that anti-TNFa therapy leads to a different autoantibody formation. Occurrence and concentrations of autoantibodies increase during the biological treatment, but these autoantibodies can present without autoimmune clinical manifestations [5]. Induction of different autoantibodies by infliximab could be explained by the fact that modulation of humoral immunity may be a more general feature of infliximab 
treatment, and this phenomenon is probably not disease specific.

In aPS/PT, a different tendency was found: more serious clinical condition was connected with higher and more frequent aPS/PT antibodies, whereas anti-TNFa treatment was followed by their significant decrease. Based on this observation we hypothesize that aPS/PT are independent autoantibodies different from "classic" aPL, and their roles in immunopathological cascades could be different. The mechanisms by which aPS/PT take part in inflammatory processes remain unclear. Nojima et al. [19, 20] has speculated that aPS/PT antibodies inhibit thrombin-mediated endothelial cell prostacyclin release, inhibit protein $\mathrm{C}$ activation, activate endothelial cells, induce pro-coagulant substances via prothrombin, and enhance platelet activation. In other words, aPS/PT can take part in the extensive cross-communication between inflammatory activity and coagulation, and their positivity could be, in this point of view, simply a consequence of sustaining inflammation.

Surprisingly, we have even more aPS/PT IgG positive samples in the ATI-positive group of patients. Immunogenicity of infliximab belongs to the emerging issues connected with the treatment with this anti-TNFa blocker. Formation of ATI can lead to limited treatment efficacy and, moreover, ATI may induct acute and/or late postinfusion reactions [21, 22]. ATI formation is another manifestation of the activity of immunopathological cascades. Predominance of aPS/PT IgG positivity in ATIpositive samples could be another evidence of assuming aPS/PT as an epiphenomenon of the inflammatory processes.

Our aPS/PT-positive patients did not experience clinically verified vascular thrombosis or other clinical signs of antiphospholipid syndrome. We realize that positivity of autoantibodies can be asymptomatic for a long time, and in definite circumstances, the clinical manifestation of the disease can occur. Our aPS/PT-positive IBD patients should be considered as at higher risk in the future, especially in the case of acute IBD flare-ups. Moreover, microvascular injury with fewer dramatic clinical signs must be considered in sustaining inflammation [23].

The current study has some limitations. Firstly, this was a retrospective study conducted on serum samples from the IBD blood bank. Therefore, we have not examined lupus anticoagulant in our cohort. It is known that aPS/PT are strongly correlated with the presence of lupus anticoagulant activity [24], and phospholipid-dependent clotting tests could be beneficial for the thrombosis risk assessment. Moreover, no data about antinuclear, anti-dsDNA and other organ non-specific autoantibodies in our cohorts are available, even though they could support or disprove proposed theory about aPS/PT as an epiphenomenon of the inflammatory processes. Future research could further test the adequacy of this hypothesis. Thirdly, this is a pilot study, so that the sample size and the restriction of the data to one medical center only limit the generalisability of the results. Our findings are associative and cause and effect cannot be determined. However, the results have biological plausibility, and provide a theory about the differences in aPS/PT and aPL behavior during the biological treatment with anti-TNFa agents.

\section{Conclusions}

A high incidence of aPS/PT was confirmed in IBD patients with active disease, with successive decreasing of autoantibody concentrations during disease healing. Unlike aPL and other autoantibodies, aPS/PT are not induced by infliximab. Thus, aPS/PT could be considered as an epiphenomenon of inflammation with proposed links between immunopathological inflammatory cascades and coagulation.

Acknowledgments Supported by Grant GAUK69810 from Charles University in Prague and by RVO VFN 64165 project from the Czech Ministry of Health.

Conflict of interest Authors declare that there is no conflict of interest that would prejudice the impartiality of this scientific work.

\section{References}

1. D'Haens GR, Panaccione R, Higgins PD, Vermeire S, Gassull M, Chowers Y et al (2011) The London Position Statement of the World Congress of Gastroenterology on Biological Therapy for IBD with the European Crohn's and Colitis Organization: when to start, when to stop, which drug to choose, and how to predict response? Am J Gastroenterol 106(2):199-212

2. Atzeni F, Turiel M, Capsoni F, Doria A, Meroni P, Sarzi-Puttini P (2005) Autoimmunity and anti-TNF-alpha agents. Ann N Y Acad Sci 1051:559-569

3. Atzeni F, Sarzi-Puttini P (2008) Autoantibody production in patients treated with anti-TNF-alpha. Expert Rev Clin Immunol 4(2):275-280

4. Ramos-Casals M, Roberto-Perez-Alvarez, Diaz-Lagares C, Cuadrado MJ, Khamashta MA, BIOGEAS Study Group (2010) Autoimmune diseases induced by biological agents: a doubleedged sword? Autoimmun Rev 9(3):188-193

5. Ramos-Casals M, Brito-Zerón P, Soto MJ, Cuadrado MJ, Khamashta MA (2008) Autoimmune diseases induced by TNFtargeted therapies. Best Pract Res Clin Rheumatol 22(5):847-861

6. Zitomersky NL, Verhave M, Trenor CC (2011) Thrombosis and inflammatory bowel disease: a call for improved awareness and prevention. Inflamm Bowel Dis 17(1):458-470

7. Nguyen G (2012) Treatment of Thromboembolism in IBD: more questions than answers. Inflamm Bowel Dis 18(1):172-173

8. Miehsler W, Reinisch W, Valic E, Osterode W, Tillinger W, Feichtenschlager $\mathrm{T}$ et al (2004) Is inflammatory bowel disease an independent and disease specific risk factor for thromboembolism? Gut 53(4):542-548 
9. Freeman HJ (2008) Venous thromboembolism with inflammatory bowel disease. World J Gastroenterol 14(7):991-993

10. Yoshida H, Granger DN (2009) Inflammatory bowel disease: a paradigm for the link between coagulation and inflammation. Inflamm Bowel Dis 15(8):1245-1255

11. Petitpain N, Gambier N, Wahl D, Chary-Valckenaere I, Loeuille D, Gillet P, French Network of Pharmacovigilance Centers (2009) Arterial and venous thromboembolic events during antiTNF therapy: a study of 85 spontaneous reports in the period 2000-2006. Biomed Mater Eng 19(4-5):355-364

12. Jaekel HP, Trabandt A, Schmid D, Grobe N, Müller EW, Ziutelis $\mathrm{V}$ et al (2004) Autoantibodies to prothrombin and phosphatidylserine/prothrombin-complexes: do they contribute to the serodiagnosis of primary and secondary anti-phospholipid syndrome? Clin Lab 50(5-6):295-304

13. Žigon P, Ambrožič A, Čučnik S, Kveder T, Rozman B, Božič B (2011) Modified phosphatidylserine-dependent antiprothrombin ELISA enables identification of patients negative for other antiphospholipid antibodies and also detects low avidity antibodies. Clin Chem Lab Med 49(6):1011-1018

14. Jaskowski TD, Wilson AR, Hill HR, Branch WD, Tebo AE (2009) Autoantibodies against phosphatidylserine, prothrombin and phosphatidylserine-prothrombin complex: identical or distinct diagnostic tools for antiphospholipid syndrome? Clin Chim Acta 410(1-2):19-24

15. Meroni PL, Borghi MO, Raschi E, Tedesco F (2011) Pathogenesis of antiphospholipid syndrome: understanding the antibodies. Nat Rev Rheumatol 7(6):330-339

16. Silverberg MS, Satsangi J, Ahmad T, Arnott ID, Bernstein CN, Brant SR et al (2005) Toward an integrated clinical, molecular and serological classification of inflammatory bowel disease: Report of a Working Party of the 2005 Montreal World Congress of Gastroenterology. Can J Gastroenterol 19:5-36
17. Vande Casteele N, Ballet V, Van Assche G, Rutgeerts P, Vermeire S, Gils A (2012) Early serial trough and antidrug antibody level measurements predict clinical outcome of infliximab and adalimumab treatment. Gut 61(2):321

18. Rutgeerts P, Vermeire S, Van Assche G (2010) Predicting the response to infliximab from trough serum levels. Gut 59(1):7-8

19. Nojima J, Kuratsune H, Suehisa E, Kitani T, Iwatani Y, Kanakura Y (2004) Strong correlation between the prevalence of cerebral infarction and the presence of anti-cardiolipin/beta2-glycoprotein I and anti-phosphatidylserine/prothrombin antibodies-Co-existence of these antibodies enhances ADP-induced platelet activation in vitro. Thromb Haemost 91(5):967-976

20. Nojima J, Iwatani Y, Suehisa E, Kuratsune H, Kanakura Y (2006) The presence of anti phosphatidylserine/prothrombin antibodies as risk factor for both arterial and venous thrombosis in patients with systemic lupus erythematosus. Haematologica 91(5): 699-702

21. Hanauer SB, Wagner CL, Bala M, Mayer L, Travers S, Diamond $\mathrm{RH}$ et al (2004) Incidence and importance of antibody responses to infliximab after maintenance or episodic treatment in Crohn's disease. Clin Gastroenterol Hepatol 2(7):542-553

22. Hanauer SB, Feagan BG, Lichtenstein GR, Mayer LF, Schreiber S, Colombel JF, ACCENT I Study Group (2002) Maintenance infliximab for Crohn's disease: the ACCENT I randomised trial. Lancet 359(9317):1541-1549

23. Sakai Y, Atsumi T, Ieko M, Amengual O, Furukawa S, Furusaki A et al (2009) The effects of phosphatidylserine-dependent antiprothrombin antibody on thrombin generation. Arthritis Rheum 60(8):2457-2467

24. Atsumi T, Koike T (2010) Antiprothrombin antibody: why do we need more assays? Lupus 19(4):436-439 